\title{
Detection of Partially Occluded Faces Using Convolutional Neural Networks
}

\author{
Chethana $\mathrm{HT}^{1,2, *}$, Trisiladevi C Nagavi ${ }^{3}$, Shashank M Athreya ${ }^{4}$ \\ ${ }^{1,3}$ Department of Computer Science \& Engineering, JSS Science \& Technology University, Mysuru \\ ${ }^{2,4}$ Department of Computer Science \& Engineering, Vidyavardhaka College of Engineering, Mysuru \\ *Corresponding author. Email: chethanaht@vvce.ac.in
}

\begin{abstract}
Partial occlusion in the face refers to the local region of the face with objects such as sunglasses, scarf, hands and beard which leads to loss of information thereby affecting the overall recognition accuracy. It is one of the challenging problems in computer vision. There are many traditional perceptions based models which have become perfect vehicles for identifying partially occluded facial images in unconstrained environments but they fail to be recognized in constrained environments. The images captured under low lighting conditions and noisy situations are called facial images with a constrained environment. The main contribution of this paper is to recognize partially occluded faces using Convolutional Neural Networks $(\mathrm{CNN})$ in a constrained environment. Hence, an attempt is made in this direction to improve the recognition accuracy for partially occluded facial images. Experimental results demonstrated that the proposed system provides a confidence level of $93 \%$ and it outperforms the state of art with the other existing partially occluded face recognition algorithms.
\end{abstract}

Keywords: Convolutional Neural Networks (CNN), Disguised Faces in the Wild (DFW), Face Landmark Estimation, Image Encodings, Partial Occlusion.

\section{INTRODUCTION}

Face recognition is one of the emerging technologies in the field of computer vision and forensics science. Partial Occlusion means interfering with the view of an object [1]. Natural and synthetic are the two types of occlusion. In natural occlusion, obstruction exists between two objects without any intention. But in synthetic occlusion, objects are covered intentionally with any rectangular box. Partial occlusion can be found in many areas like iris recognition, ear recognition, face recognition and medical image processing. Presence of partially occluded features in face is one of the challenging problems that exist in face recognition.

Partial occlusion in the face refers to the local region of the face covered with objects such as sun glasses, scarf, hands and beard. The presence of partial occlusion features in the face is shown from Figure 1 to Figure 3 and it reduces the overall performance of the face detection systems. Therefore, robustness to partial occlusion in face has become a very crucial task in today's scenario.

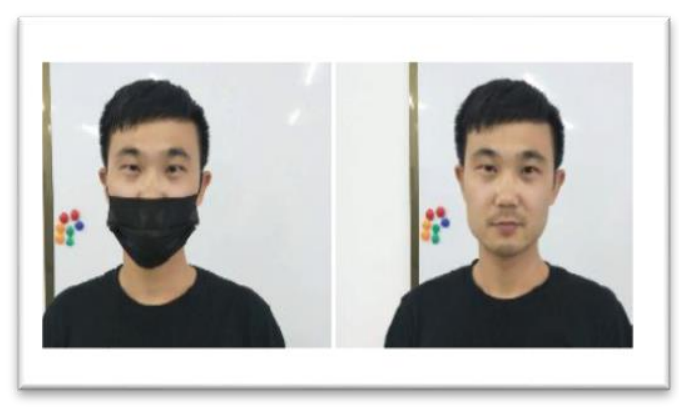

Figure 1 Partial Occlusion by Mask [2]

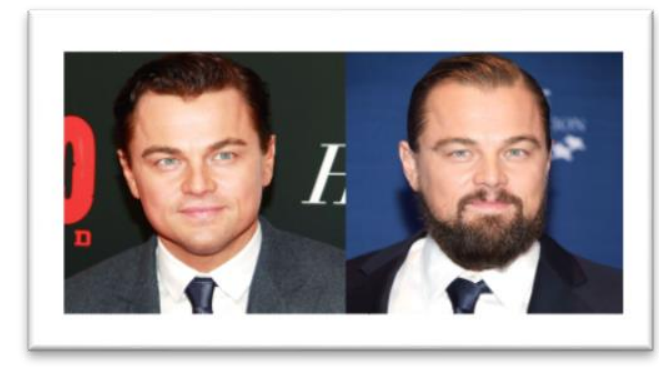

Figure 2 Partial Occlusion by Beard [2] 


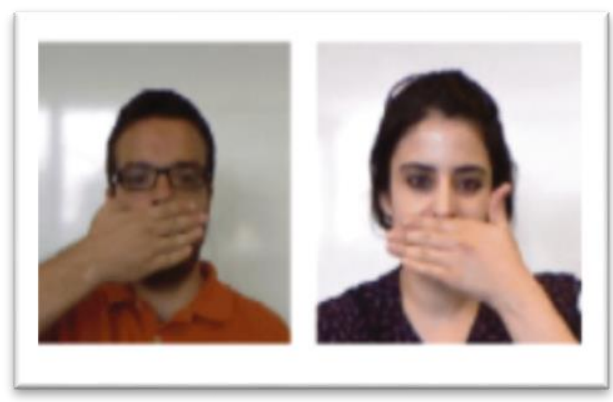

Figure 3 Partial Occlusion by Hand [2]

Over the past few years, identification of partially occluded faces has drawn attention of the research community. Researchers are contributing and observing better results, but there are still many opportunities for improvement with the occluded faces. Due to recent advancements in computer technology, people change their looks and commit crime. If an individual changes his looks either by face occluded by hand, beard or mask it will affect the overall performance of the system.

In the field of forensics, if an individual has committed a crime having partially occluded features then it is a very difficult task to identify the criminal. Detection of partially occluded facial images can be considered as one of the significant applications in the field of forensics. Therefore, there is a need for development of automated partially occluded facial recognition systems to identify the criminals in an efficient way.

Traditional Neural Network based models are unable to capture the patterns of occlusion effectively and they find it difficult to recognize partially occluded facial images. But CNN learns the features automatically by extracting the correct and relevant features from the input image. It captures only the spatial features which in turn help to identify the image accurately along with the location of the image and relationship with the other objects in the image.

Hence in the proposed system, an attempt is made in this direction to detect partially occluded facial images using $\mathrm{CNN}$ in a constrained environment.

The main objectives of this research work are stated below.

1. Development of an automated partially occluded facial recognition system.

2. Exhaustive experimental analysis of the proposed system.

3. Comparison of the results with the state of the art partially occluded face recognition algorithms.

The research paper is organized as follows. Section 1 and Section 2 present introduction and literature review on partially occluded facial images respectively. A detailed discussion on the proposed system is presented in section 3. Experimental results and discussion is presented in section 4. Finally, section 5 portrays the concluding remarks.

\section{RELATED WORKS}

Literature survey reveals that faces can be recognized in both restricted and unrestricted environments. In a real time, scenario, a lot of challenges exist to solve the problem of partially occluded faces [1]. Henceforth, many authors have proposed different techniques to rectify the problem of partially occluded faces.

Zhang et al. [3] presented an approach for a part based visual tracking of three different frames in video. Experimental results prove that performance of this approach is better when compared with other visual tracking methods. In another work, Kortylewski et al. [4] developed an approach based on the combination of deep neural networks with the composition models. The model is able to recognize the occluded objects even if the occluded objects are not trained.

Similarly, a probabilistic framework for pedestrian detection with occlusion handling is proposed by Ouyang et al. [5]. Experiments are carried out on three public datasets and CUHK occlusion datasets. This framework performs better than the occluded pedestrian data. Hou $e t$ al. [6] presented an approach based on Spatio -Temporal Completion network (STCnet) which addresses the problem of the partial occlusion. The occluded parts in the video are recovered using the STCnet framework. Both recovered regions and uncovered regions are combined and then fed into training.

In another work [7], compositional models are combined with Deep Convolutional Neural Networks (DCNN) which is used to differentiate between real partial and artificially occluded images. Experimental results on MS-COCO dataset envisaged that proposed approach out performs the DCNN by classifying the object as partially occluded even if not trained. Zaman $e t$ al. [8] proposed a strategy based on local lateral subspace which is used to extract the local features. Experiments are carried out on three datasets and research has yielded $90.70 \%$ recognition accuracy.

A dissimilarity function for partial occlusion in the case based reasoning (CBR) system was developed by Saha et al. [9] which will discard the features from partially occluded regions. This approach provides a better performance in uncontrolled lighting conditions. Another team of researchers Priya et al. [10] presented an approach based on novel occlusion invariant face recognition. It consisted of occlusion based detection phase and mean based weight matrix face recognition phase. Proposed approach shows superior performance compared with other conventional approaches. Another novel method is proposed by Tarres et al. [11] which 
uses the Lophoscopic Principal Component Analysis (PCA) method. Experimental results envisaged-that performance of the proposed approach is improved when compared to PCA.

To solve the problem of partial occlusion, Bommidi et al. [12] proposed a novel approach based on a combination of compressed string matching algorithm with run length encoding. The proposed approach is experimented on Extended Yale-B and AR face datasets. It shows significant performance in terms of recognition accuracy and speed. Sreelakshmi et al. [13] proposed an approach based on MobileNet architecture of CNN for handling partially occluded facial images. The model achieved an accuracy of $92.5 \%$ on partial occluded facial images.

Zou et al. [14] proposed an approach to reconstruct the remaining part of the faces from partially occluded faces. AlexNet, a pre-training model is used for training normal face images and tested with occluded face images. Experimental results prove that performance of classification has improved effectively. A Sparse representation algorithm [15] for fusion error is developed by $\mathrm{Li}$ which provided a new solution for identifying partially occluded facial images.

From the literature survey, it is observed that the majority of the work is available in the field of unconstrained environment [20-25] especially in different areas of partial occlusion [1,2,4]. Limited work is reported in the area of partial occlusion in face recognition with constrained environment and achieved a recognition accuracy of $92.38 \%$. As a result, identifying the partially occluded facial images has drawn attention of many researchers. There exists a scope for the development of new approaches to improve the recognition accuracy in the field of partial occlusion [2630] with a constrained environment.

\section{PROPOSED SYSTEM}

Proposed system consists of four steps which are built in Python using OpenFace and dlib. They are Face Detection, Projection of Faces, Encoding Faces and identifying the person from image encodings. The architecture of the partially occluded facial recognition system is shown in Figure 4.

\subsection{Preprocessing}

It is the first step in proposed system. An input image is converted into black and white because color data is not required to find the faces. Every pixel of an input image is analyzed. For every pixel, the pixels that are adjacent to it are identified and compared with the directional flow of luminosity between them [16].

An arrow is drawn to the darker image representing the direction. This process is repeated for every pixel in the image and an arrow is replaced by each pixel. Flow of this process takes place from lighter to darker regions across the entire image. This process is known as gradients.

Divide the image into small squares of $16 \times 16$ pixels each. In each square, count the number of gradient points in each significant direction. Replacement process takes place by replacing a square in the image with the arrow directions that were the strongest. After this process, the original image is converted into a straightforward representation thereby capturing the basic structure of a face.

\subsection{Face Detection}

Face detection is the next step in the proposed system. Location of the faces in an input image is identified so that it can convey that they are apart. Later area of the face in the image is calculated and it is further passed to the next step.

In the proposed system, Convolutional Neural Networks $(\mathrm{CNN})$ is used to detect all the faces in the image.

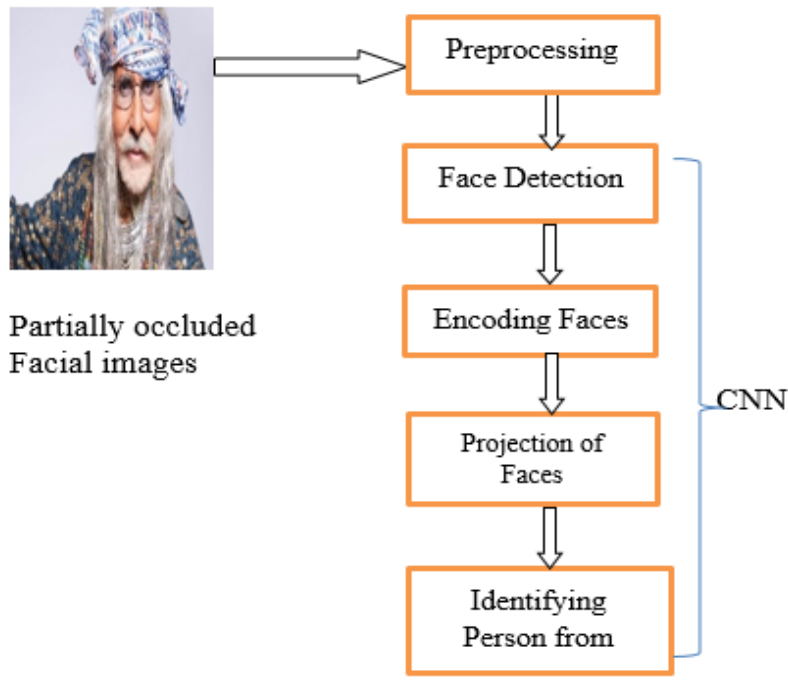

Figure 4 Architecture of partially occluded facial recognition system

\subsection{Projection of Faces}

In this step, wrapping process takes place for each and every detected in such a way that the eyes and lips are always at the same location in the image Face landmark estimation algorithm is used to carry out this process. Then CNN is trained to identify the 68 specific points on every face [31-36].

The mean normalized distance (MND) is used to extract 68 specific points on every face which is calculated is calculated using the Equation (1). 
$\operatorname{MND}=\left(1 / 68 \sum_{i=1}^{68} \quad \frac{\sqrt{(a-b)^{2}}}{\mathrm{~W}}+\frac{\sqrt{(m-n)^{2}}}{H}\right)$

Here $\mathrm{W}$ denotes width, $\mathrm{H}$ denotes height, $\mathrm{a}, \mathrm{b}, \mathrm{m}$ and $\mathrm{n}$ are the variables used to calculate the distance between pixels.

\subsection{Encoding of Faces}

Comparing the unknown faces with all the faces of the people stored in the database becomes an easy task for smaller datasets. If the size of the dataset is large, then it becomes a very difficult and challenging task to loop through every image and identify the person within a few milliseconds.

To overcome this situation, a few essential measurements from each face are extracted so that it can be used to measure the unknown face accurately. As a result, in the proposed system, $\mathrm{CNN}$ is trained to generate face encodings. Once the network is trained, it will generate 128 measurements (face encodings) for any given partially occluded facial image.

\subsection{Identifying the person from image encodings}

It is the last step in the proposed system. Here in order to identify the person in the database, the trained classifier will generate a face encoding for the given input image. The generated face encodings is compared with the face encodings already stored in the database. The closest match will be retrieved for the given input image.

During this process, if multiple matches for the same person occur, then the people in the photos look very similar. To make the face comparison process stricter, tolerance levels are set to low. In this regard, tolerance levels are adjusted so that lower the tolerance levels, the better the accuracy for the recognition of partially occluded facial images.

\section{EXPERIMENTAL RESULTS AND DISCUSSION}

In this section, experiments carried out on Disguised Faces in the Wild dataset [22] are discussed. DFW dataset is collected from Image Analysis and Biometrics Lab from IIIT Delhi which consists of 1000 subjects with a total of 1,11,157 images. For experimentation purpose, 400 samples are used for training and 600 samples are used for testing. The statistics of dataset samples are indicated in the Table 1.

Table 1. Statistics of dataset samples [22]

\begin{tabular}{|c|c|c|c|c|}
\hline \multirow[b]{2}{*}{$\begin{array}{c}\text { Dataset } \\
\text { Name }\end{array}$} & \multicolumn{2}{|c|}{ Number of Subjects } & \multirow[b]{2}{*}{$\begin{array}{c}\text { Challeng } \\
\text { es }\end{array}$} & \multirow[b]{2}{*}{ Source } \\
\hline & Testing & Training & & \\
\hline
\end{tabular}

\begin{tabular}{|l|l|l|l|l|}
\hline Disguised & 600 & 400 & Normal, & Image \\
Faces in & & & Validatio & Analysis \\
Wild(DF & & n, & and \\
W) & & & Disguised & Biometric \\
& & & and & s Lab@ \\
& & & imperson & IIIT \\
& & & ator & Delhi \\
& & & images & \\
\hline
\end{tabular}

The results of the experiments conducted on DFW dataset are shown from the Figure 5 to Figure 8.
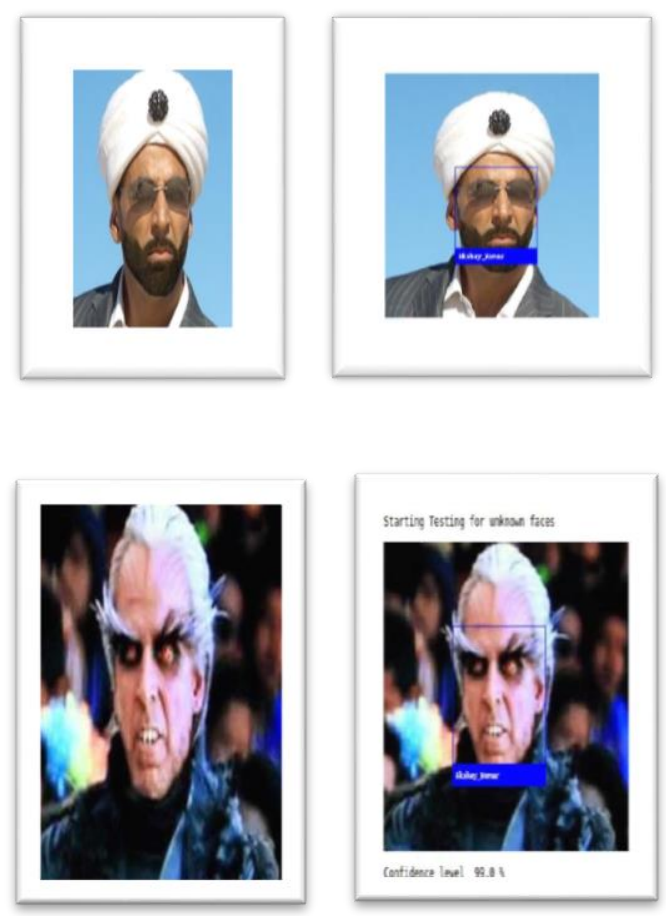

Figure 5 Partially Occluded Face Recognition of Akshay Kumar
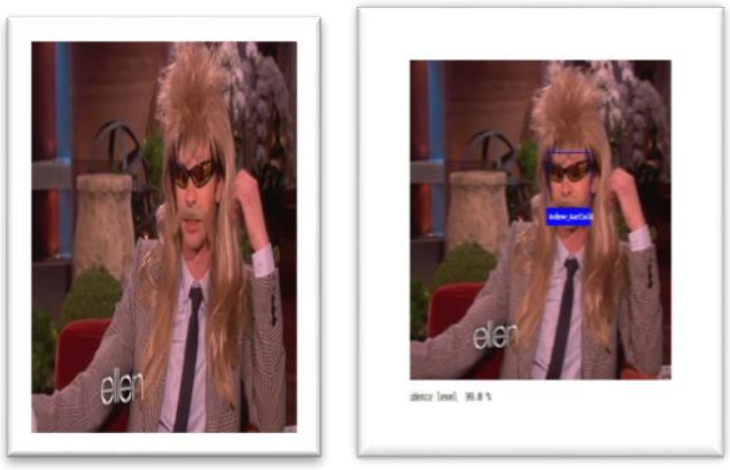

Figure 6 Partially Occluded Face Recognition of Andrew Garfield 

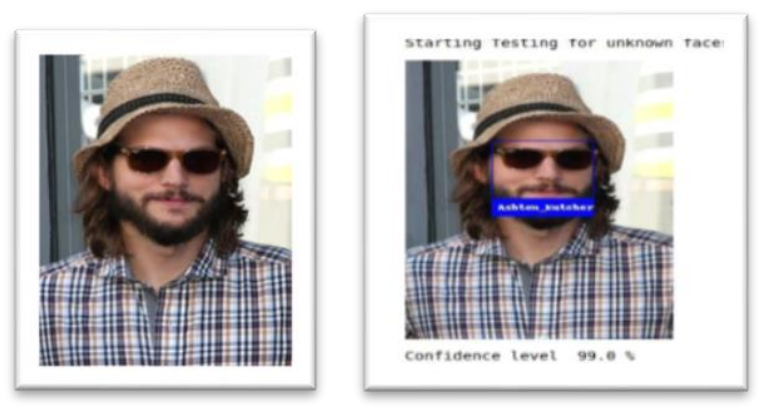

Figure 7 Partially Occluded Face Recognition of Ashton Kutcher

Confidence levels (accuracy) are the likelihood of the results being true for the total population and expressed in percentage form. It is one of the measures used in the proposed system to recognize partially occluded faces. Out of 1000 samples, 930 samples were correctly recognized and 70 samples were incorrectly recognized.

Accuracy is one of the metrics used to evaluate the performance of a proposed system. It is calculated using the Equation (2).

Accuracy $=\frac{\text { Total number of samples correctly recognized }}{\text { Total number of samples }}$
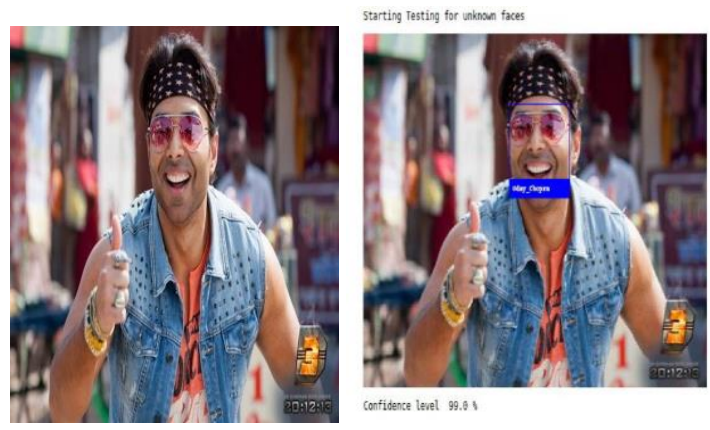

Figure 8 Partially Occluded Face Recognition of Uday Chopra

Proposed system provides a confidence level of $93 \%$ on DFW dataset. The proposed system is compared with other existing approaches for partially occluded facial images are illustrated in Table 2.

Figure 9 illustrates the graphical representation of comparative study. In this plot, a value in Y-axis denotes the recognition accuracy and a value in $\mathrm{X}$-axis denotes the name of existing approaches and proposed system.

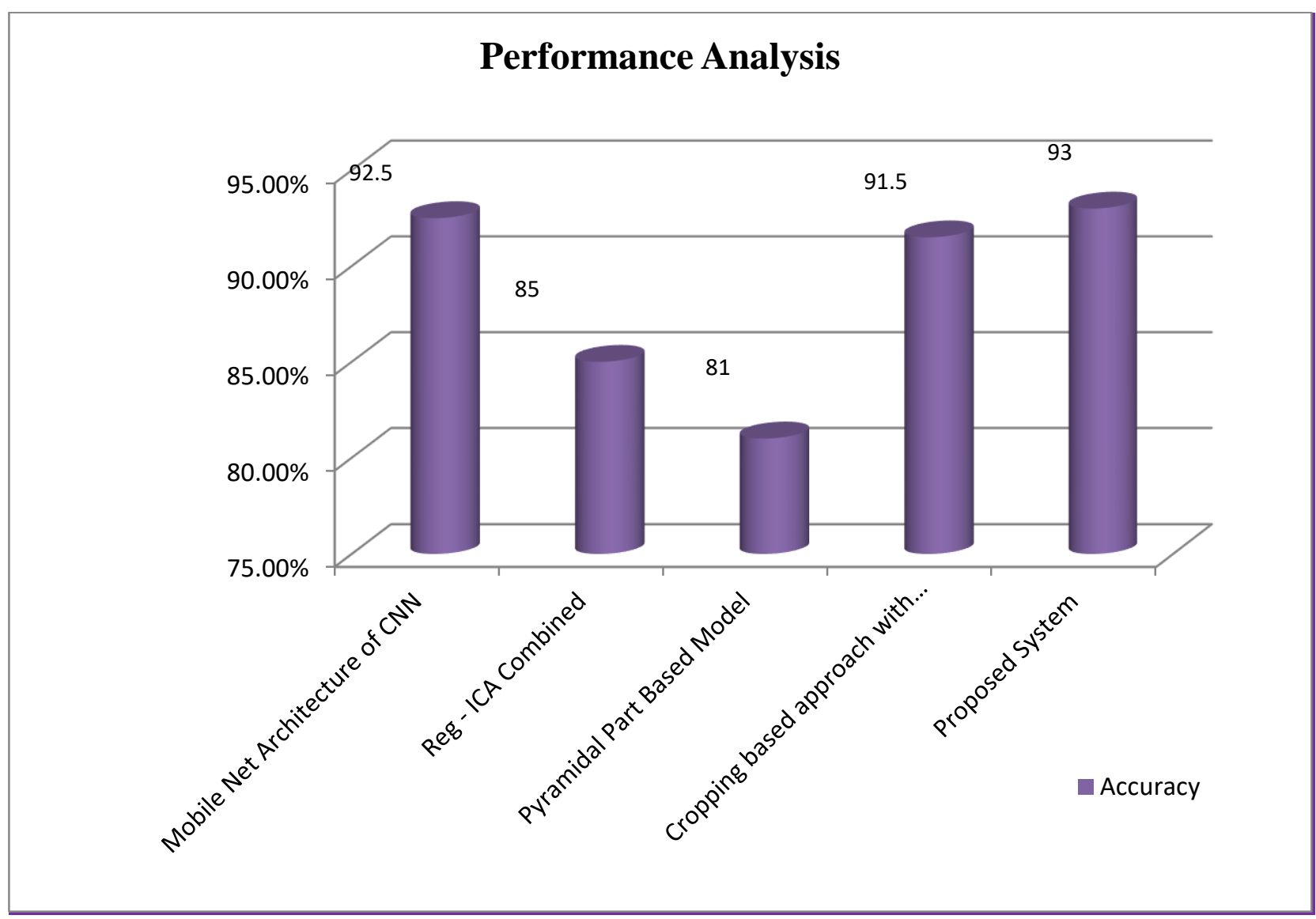

Figure 9 Graphical representation of comparative study between proposed system and existing approaches with reference to accuracy. 
Table 2. Comparative study of proposed system with other existing approaches for partially occluded facial images.

\begin{tabular}{|c|c|c|c|}
\hline Sl. No & Authors & Methodology & Recognition Accuracy \\
\hline 1 & Lakshmi et al. [13] (2019) & Mobile Net Architecture of CNN & $92.5 \%$ \\
\hline 2 & Maghari et al. [ 17] (2020) & $\begin{array}{l}\text { Region Independent Component Analysis(Reg- ICA } \\
\text { Combined ) }\end{array}$ & $85 \%$ (Sunglass) \\
\hline 3 & Thu et al. [18] (2020) & Pyramidal Part Based Model & $\begin{array}{l}81 \% \text { (Prince of Songkla } \\
\text { University dataset) }\end{array}$ \\
\hline 4 & Li et al. [19] (2021) & $\begin{array}{l}\text { Cropping based approach with Convolutional Block } \\
\text { Attention Module }\end{array}$ & $\begin{array}{l}91.5 \% \\
\text { (Masked Web Face dataset) }\end{array}$ \\
\hline 5 & Proposed System & CNN with Face Encodings & $\begin{array}{l}\text { 93\% (Confidence } \\
\text { Level) }\end{array}$ \\
\hline
\end{tabular}

From the comparative study, it is observed that the proposed system provides an appreciable recognition accuracy of $93 \%$. It outperforms compared to the existing partially occluded face recognition algorithms.

In the proposed system, the partially occluded facial images are fed into $\mathrm{CNN}$ for training which automatically learns the input images through filters thereby decreasing the burden of learning. It always captures the spatial features which will detect the partially occluded facial images accurately. Hence, the proposed system performs better than existing algorithms.

\section{CONCLUSION}

The problem of partial occlusion in face is studied relatively less when compared to normal face recognition having challenges like pose variation, illuminations and expression variations. An attempt is made in this direction, to recognize partially occluded faces using $\mathrm{CNN}$. The proposed system is evaluated on the DFW dataset which provides an appreciable recognition accuracy of $93 \%$. The limitation of this research work is that the performance of the proposed system can still be improved by testing on larger datasets. Since the nature of occlusion on the face vary greatly, a lot of new approaches can be developed for automating partially occluded face recognition algorithms.

\section{ACKNOWLEDGMENTS}

Authors would like to thank the researchers V. Kushwaha, M. Singh, R. Singh, M. Vatsa, N. Ratha, and R. Chellappa, A. [22] for providing their dataset (Disguised Faces in the Wild) to carry out the experimentation.

\section{REFERENCES}

[1] Aisha Azeem, Muhammad Sharif, Mudassar Raza, Marryam Murtaza, A Survey: Face Recognition Techniques under Partial Occlusion, International Arab Journal of Information Technology, January 2014.
[2] J. Y. Ramìrez Cornejo, H. Pedrini, Emotion Recognition from Occluded Facial Expressions Using Weber Local Descriptor, 25th International Conference on Systems, Signals and Image Processing (IWSSIP), Maribor, 2018.

[3] Tianzhu Zhang, Kui Jia, Changsheng Xu, Yi Ma, Narendra Ahuja, Partial Occlusion Handling for Visual Tracking via Robust Part Matching, IEEE Conference on Computer Vision and Pattern Recognition, June 2014,Columbus,OH,USA,DOI:10.1109/CVPR.2014.164.

[4] Adam KortyLewski, Qing Liu,Huiyu Wang, Zhishuai Zhang, Alan Yuille, Combining Compositional Models and Deep Networks for Robust Object Classification under occlusion, arXiv:1905.11826v4 [cs.CV] 29, January 2020 .

[5] Wanli Ouyang, Xingyu Zeng, Xiaogang Wang, Partial Occlusion Handling in Pedestrian Detection with a Deep Model, Journal Of Latex Class Files, Vol. 11, No. 4, December 2012.

[6] Ruibing Hou, Bingpeng Ma, Hong Chang, Xinqian Gu, Shiguang Shan, Xilin Chen, VRSTC: Occlusion-Free Video Person Re-Identification, arXiv:1907.08427v1 [cs.CV] 19, July 2019.

[7] Adam Kortylewski, Ju He, Qing Liu, Alan Yuille, Compositional Convolutional Neural Networks: A Deep Architecture with Innate Robustness to Partial Occlusion, arXiv: 2003.04490 v3 [cs.CV] 29, April 2020.

[8] Fadhlan Kamaru Zaman Amir Akramin Shafie, Yasir Mohd Mustafah, International Journal of Automation and Computing, 13(4), 319-337, DOI: 10.1007/s11633-0160974-6, August 2016.

[9] David W Saha, Jean Lieber (Eds.), Case-Based Reasoning Research and Development, 25 ${ }^{\text {th }}$ International Conference, ICCBR 2017 Trondheim, Norway, June 2628, 2017.

[10] G Nirmala Priya, R S D Wahida Banu, Occlusion invariant face recognition using mean based weight matrix and support vector machine, Vol. 39, pp. 303-315, Indian Academy of Sciences, April 2014.

[11] Francesc Tarrés, Antonio Rama, A Novel Method for Face Recognition under partial occlusion or facial expression Variations, ELMAR, 47 $7^{\text {th }}$ International Symposium,2005 DOI: 10.1109/ELMAR.2005.193667. 
[12] Bommidi K, Sundaramurthy S, A compressed string matching algorithm for face recognition with partial occlusion, Multimedia Systems.27, 191-203, https://doi.org/10.1007/s00530-020-00727-9, 2021.

[13] Sreelakshmi P, Sumithra M D, Facial Expression Recognition robust to partial Occlusion using MobileNet, International Journal of Engineering Research \& Technology, Vol. 08, Issue 06, June 2019.

[14] Min Zou, Mengbo You, Takuya Akashi Reconstruction of Partially Occluded Facial Image for Classification,https://doi.org/10.1002/tee.23335, February 2021.

[15] Zijun Li, Sparse representation algorithm for fusion error, Journal of Physics: Conference Series, doi:10.1088/17426596/1871/1/012120, 2021.

[16] Zaman, Fadhlan, Shafie, A.A, Mohd Mustafah, Yasir, Robust face recognition against expressions and partial occlusions, International Journal of Automation and Computing. 13. 10.1007/s11633-016-0974-6, 2016.

[17] Ashraf Y. A. Maghari (2020): Recognition of partially occluded faces using regularized ICA, Inverse Problems in Science and Engineering, DOI: 10.1080/17415977.2020.1845329, November 2020.

[18] M Thu, N. Suvonvorn, Pyramidal Part-Based Model for Partial Occlusion Handling in Pedestrian Classification, Hindawi Advances in Multimedia, Volume 2020, Article ID 6153580, $15 \quad$ pages, https://doi.org/10.1155/2020/6153580, February 2020.

[19] Yande Li , Kun Guo, Yonggang Lu , Li Liu, Cropping and attention based approach for masked face Recognition, Applied Intelligence (2021) 51:3012-3025, https://doi.org/10.1007/s10489-020-02100-9,February 2021.

[20] Shashank M Athreya, Shreevari S P, Siddesh Aradhya BS, Sandeep Kiran and Chetana H T, A Survey on partially occluded faces , Evolutionary Computing and Mobile Sustainable Networks, Proceedings of ICECSMN pp 61-67, 2020.

[21] Chethana H T, Trisiladevi C Nagavi, Face Recognition for criminal analysis using Haar Classifier, i-manager's Journal on Computer Science,Vol.8, No.1, 2020.

[22] V. Kushwaha, M. Singh, R. Singh, M. Vatsa, N. Ratha, and R. Chellappa, Disguised Faces in the Wild, IEEE International Conference on Computer Vision and Pattern Recognition Workshop on Disguised Faces in the Wild, 2018.

[23] Parameshachari, B. D., H. T. Panduranga, and Silvia liberata Ullo. "Analysis and computation of encryption technique to enhance security of medical images." In IOP Conference Series: Materials Science and Engineering, vol. 925, no. 1, p. 012028. IOP Publishing, 2020.

[24] Rajendran, Ganesh B., Uma M. Kumarasamy, Chiara Zarro, Parameshachari B. Divakarachari, and Silvia L. Ullo. "Land-use and land-cover classification using a human group-based particle swarm optimization algorithm with an LSTM Classifier on hybrid preprocessing remote-sensing images." Remote Sensing 12, no. 24 (2020): 4135.

[25] Seyhan, Kübra, Tu N. Nguyen, Sedat Akleylek, Korhan Cengiz, and SK Hafizul Islam. "Bi-GISIS KE: Modified key exchange protocol with reusable keys for IoT security." Journal of Information Security and Applications 58 (2021): 102788.

[26] H. Li, K. Yu, B. Liu, C. Feng, Z. Qin and G. Srivastava, "An Efficient Ciphertext-Policy Weighted AttributeBased Encryption for the Internet of Health Things," IEEE Journal of Biomedical and Health Informatics, 2021, doi: 10.1109/JBHI.2021.3075995.

[27] L. Zhen, A. K. Bashir, K. Yu, Y. D. Al-Otaibi, C. H. Foh, and P. Xiao, "Energy-Efficient Random Access for LEO Satellite-Assisted 6G Internet of Remote Things", IEEE Internet of Things Journal, doi: 10.1109/JIOT.2020.3030856.

[28] Nguyen, Tu N., Bing-Hong Liu, Nam P. Nguyen, and Jung-Te Chou. "Cyber security of smart grid: attacks and defenses." In ICC 2020-2020 IEEE International Conference on Communications (ICC), pp. 1-6. IEEE, 2020.

[29] Bhuvaneswary, N., S. Prabu, K. Tamilselvan, and K. G. Parthiban. "Efficient Implementation of Multiply Accumulate Operation Unit Using an Interlaced Partition Multiplier." Journal of Computational and Theoretical Nanoscience 18, no. 4 (2021): 1321-1326.

[30] L. Tan, N. Shi, K. Yu, M. Aloqaily, Y. Jararweh, "A Blockchain-Empowered Access Control Framework for Smart Devices in Green Internet of Things", ACM Transactions on Internet Technology, vol. 21, no. 3, pp. 120, 2021,https://doi.org/10.1145/3433542.

[31] Z. Guo, A. K. Bashir, K. Yu, J. C. Lin, Y. Shen, "Graph Embedding-based Intelligent Industrial Decision for Complex Sewage Treatment Processes", International Journal of Intelligent Systems , 2021 doi: $10.1002 /$ int.22540

[32] Bhuvaneswary, N., S. Prabu, S. Karthikeyan, R. Kathirvel, and T. Saraswathi. "Low Power Reversible Parallel and Serial Binary Adder/Subtractor." Further Advances in Internet of Things in Biomedical and Cyber Physical Systems (2021): 151.

[33] Pham, Dung V., Giang L. Nguyen, Tu N. Nguyen, Canh V. Pham, and Anh V. Nguyen. "Multi-topic misinformation blocking with budget constraint on online social networks." IEEE Access 8 (2020): 78879-78889.

[34] K. Yu, L. Tan, X. Shang, J. Huang, G. Srivastava and P. Chatterjee, "Efficient and Privacy-Preserving Medical Research Support Platform Against COVID-19: A Blockchain-Based Approach", IEEE Consumer Electronics Magazine, doi: 10.1109/MCE.2020.3035520.

[35] Z. Guo, Y. Shen, A. K. Bashir, M. Imran, N. Kumar, D. Zhang and K. Yu, "Robust Spammer Detection Using Collaborative Neural Network in Internet of Thing 
Applications", IEEE Internet of Things Journal, vol. 8, no. 12, pp. 9549-9558, 15 June15, 2021, doi: 10.1109/JIOT.2020.3003802.

[36] Subramani, Prabu, Ganesh Babu Rajendran, Jewel Sengupta, Rocío Pérez de Prado, and Parameshachari Bidare Divakarachari. "A block bi-diagonalization-based pre-coding for indoor multiple-input-multiple-outputvisible light communication system." Energies 13, no. 13 (2020): 3466. 